CARPATHIAN J. MATH.

Volume 38 (2022), No. 1,

Pages $57-66$
Online version at https : //www. carpathian. cunbm. utcluj. ro/

Print Edition: ISSN 1584 - 2851; Online Edition: ISSN 1843 - 4401

DOI: https://doi.org/10.37193/CJM.2022.01.06

Dedicated to the memory of Academician Mitrofan M. Choban (1942-2021)

\title{
On the properties of lexicographic tolerable robust solution sets for uncertain multi-objective optimization problems
}

\author{
PORNPIMON BORIWAN ${ }^{1}$, DAISHI KUROIWA ${ }^{2}$ and NARIN PETROT ${ }^{1,3^{*}}$
}

\begin{abstract}
This study provides the important properties of the lexicographic tolerable robust solution for uncertain multi-objective optimization problems which was introduced by Boriwan et al. [Boriwan, P.; Ehrgott, M.; Kuroiwa, D.; Petrot, N. The lexicographic tolerable robustness concept for uncertain multi-objective optimization problems: a study on water resources management. Sustainability. 12 (2020), no. 18, article number 7582.]. Also, the relationship between the lexicographic tolerable solution concept and the well-known robust solution, as the set-based robust efficiency [Ehrgott, M.; Ide, J.; Schöbel, A. Minmax robustness for multi-objective optimization problems. European Journal of Operational Research. 239 (2014), no. 1, 17-31.], are provided.
\end{abstract}

\section{INTRODUCTION}

Many real-world problems are often faced with several objectives which need to be concurently optimized, but not all relevant input data are known exactly in advance. These two issues have been studied in the areas of multi-objective optimization and robust optimization, respectively. Evidently, since the combination of both robust optimization and multi-objective optimization is versatile and adaptable, recent years have seen numerous applications on the problem of management science for which problems are structured in mathematical form of robust multi-objective optimization to generate managerially relevant insights. For example, water resources management planning, as in [12], time table information systems, see $[6,8]$, and flight route planning, see in $[3,13]$, are some of the concrete applications of robust multi-objective optimization.

Due to the usefulness of robust multi-objective optimization, various approaches have been presented throughout the literatures for which practitioners can choose from those concepts to fit in the respective robust multi-objective application at their hand. However, as a lack of total order in comparing the vector-valued functions and unclear definition in the worst case for robust multi-objective optimization problems, many researchers are trying to find reliable approaches which are suitable for various practical problems. According to the worst case in minmax robustness which was firstly presented by Soyster [14] and extensively studied by Ben-Tal and Nemirovski [1] in robust single objective optimization problems, the classical concept of robust solution was introduced by Kuroiwa and Lee [10] for uncertain multi-objective optimization problems, so-called the pointbased minmax robust efficiency concept. Another interpretation of minmax robustness concept for uncertain multi-objective optimization problems is the set-based minmax robust efficiency concept which was proposed by Ehrgott et al. [4]. The idea underlying this solution concept of minmax robustness is to find solutions that minimizes the objective

Received: 29.12.2020. In revised form: 26.04.2021. Accepted: 03.05.2021

2010 Mathematics Subject Classification. 47H10, 54H25.

Key words and phrases. robust optimization, lexicographic tolerable robust solution, uncertain multiobjective optimization problem .

Corresponding author: N. Petrot; narinp@nu.ac.th 
function in the worst case of the considered uncertain multi-objective optimization problems. According to this robustness concept, the decision makers should obtain the robust solution which performs remarkably well with a good trade-off for all objective functions. Notice that the point-based minmax robust efficiency concept and the set-based minmax robust efficiency concept are identical in the case of considering the objective-wise uncertain multi-objective optimization problem. Recently, another direction of robustness concepts for uncertain multi-objective optimization problems was proposed by Boriwan et al. [2], which is called the lexicographic tolerable robust solution. This approach provides a solution which is appropriate for the practical problems in which the objective function is composed of different level priorities and the worst performance vector of the solution obtained by the proposed concept is close to the reference point of the problem, within an acceptable tolerance threshold. The main conclusion in this paper will be focused on this solution concept.

The contribution of the current paper is composed of two major sections. Firstly, we analyse and present the important properties of the lexicographic tolerable robust solution which was proposed by Boriwan et al. [2]. Secondly, we analyze the relation of the lexicographic tolerable robust solution to the existing set-based minmax robust efficiency concept on uncertain multi-objective optimization problems.

\section{PRELIMINARIES}

2.1. Notations and Basic concepts. In this section, we introduce the main notations and basic concepts which will be used throughout this work. For each $p \in \mathbb{N}$, we use an abbreviation $I_{p}$ for the index set $\{1,2, \ldots, p\}$. For vectors $x, y \in \mathbb{R}^{p}$ with $x=\left(x_{1}, x_{2}, \ldots, x_{p}\right), y=$ $\left(y_{1}, y_{2}, \ldots, y_{p}\right)$, we define notations $\precsim, \preccurlyeq, \prec$, and $\leq_{\text {lex }}$ as follows:

$$
\begin{aligned}
& x \precsim y \Leftrightarrow x_{i} \leqslant y_{i} \text { for all } i \in I_{p}, \\
& x \preccurlyeq y \Leftrightarrow x_{i} \leqslant y_{i} \text { for all } i \in I_{p} \text { and } x \neq y, \\
& x \prec y \Leftrightarrow x_{i}<y_{i} \text { for all } i \in I_{p}, \\
& x \leq \text { lex } y \Leftrightarrow x_{m}<y_{m} \text { where } m:=\min \left\{k \mid x_{k} \neq y_{k}\right\} .
\end{aligned}
$$

We may note that the notations $\precsim, \preccurlyeq, \prec$, and $\leq_{l e x}$ are used to stand for the relations on $\mathbb{R}^{p}$ and $\leqslant,<$ are used to stand for the relations on $\mathbb{R}$, respectively.

By using the analogously reversed inequalities, including $\succsim, \succcurlyeq, \succ$, and $\geq_{\text {lex }}$, we denote the orthants of $\mathbb{R}^{p}$ as follows:

$$
\begin{aligned}
& \mathbb{R}_{\succsim}^{p}:=\left\{x \in \mathbb{R}^{p} \mid x \succsim 0\right\}, \\
& \mathbb{R}_{\succ}^{\widetilde{\widetilde{p}}}:=\left\{x \in \mathbb{R}^{p} \mid x \succcurlyeq 0\right\}, \\
& \mathbb{R}_{\succ}^{\text {p }}:=\left\{x \in \mathbb{R}^{p} \mid x \succ 0\right\}, \\
& \mathbb{R}_{\geq_{\text {lex }}}^{p}:=\left\{x \in \mathbb{R}^{p} \mid x \geq_{\text {lex }} 0\right\} .
\end{aligned}
$$

For a subset $A$ of $\mathbb{R}^{p}$, by using the binary relation $\leq_{l e x}$, Boriwan et al. [2] considered the infimum of set $A$ with respect to the lexicographic order $\leq_{l e x}$, and denoted such vector by $\inf A$. That is, with lex

$$
\inf _{\text {with lex }} A \leq_{l e x} x, \text { for all } x \in A .
$$

Notice that by means of $\inf _{\text {with lex }} A$ in (2.1), Boriwan et al. [2], determined the concept of the reference point as the infimum of set of the worst performance vectors for the lexicographic tolerable robust solution concept. It is worth pointing out that the corresponding reference point is satisfied the priority levels of each component of the vectors in the considered set with respect to the concept (2.1). After introducing such lexicographic tolerable robustness approach, they also provided the method to compute an acceptable tolerance 
threshold which can be guaranteed the existence of lexicographic tolerable robust solutions. For more details on nonemptiness and the method of computing an acceptable tolerance threshold, we refer the reader to see in [2].

In order to review the concept of lexicographic tolerable robust solution in [2], we need to recall the following important notation of ordering the values nonincreasingly which will be used throughout this paper. The sort function, $\operatorname{sort}(\cdot): \mathbb{R}^{p} \rightarrow \mathbb{R}^{p}$, is a function that reordering the component of each vector on $\mathbb{R}^{p}$ in a nonincreasing way. That is, for each $y \in \mathbb{R}^{p}$,

$$
\operatorname{sort}(y):=\left(y_{\sigma(1)}, y_{\sigma(2)}, \ldots, y_{\sigma(p)}\right),
$$

where $\sigma$ is a permutation on $I_{p}$ such that $y_{\sigma(1)} \geqslant y_{\sigma(2)} \geqslant \cdots \geqslant y_{\sigma(p)}$. In this case, we will write $\operatorname{sort}(y):=\left(\operatorname{sort}_{1}(y), \operatorname{sort}_{2}(y), \ldots, \operatorname{sort}_{p}(y)\right)$.

Next section, we provide a short introduction of the lexicographic tolerable robust solution concept [2] and also the relevant notations.

2.2. Lexicographic robust solutions with respect to the tolerance threshold for uncertain multi-objective optimization problems. For an objective function $f: \mathbb{R}^{n} \times \mathcal{U} \rightarrow \mathbb{R}^{p}$, a feasible set $X \subseteq \mathbb{R}^{n}$, and an uncertainty set $\mathcal{U}=\left\{s_{1}, \ldots, s_{q}\right\}$, the uncertain multi-objective optimization problem $\mathcal{M P}(\mathcal{U})$ is given as a family of $\left\{\mathcal{M P}\left(s_{j}\right) \mid s_{j} \in \mathcal{U}\right\}$ of deterministic multi-objective optimization problems

$$
\begin{array}{ll}
\left(\mathcal{M P}\left(s_{j}\right)\right) & \min f\left(x, s_{j}\right) \\
& \text { subject to } x \in X .
\end{array}
$$

For each $i \in I_{p}$ and $x \in \mathbb{R}^{p}$, the vector which is composed of the value of the $i^{t h}$ component from each scenario of the objective function $f$ at the alternative solution $x$ is denoted by $c^{(i)}(x)$ in Boriwan et al. [2]. That is, for each $x \in \mathbb{R}^{n}$,

$$
c^{(i)}(x):=\left(f_{i}\left(x, s_{1}\right), f_{i}\left(x, s_{2}\right), \ldots, f_{i}\left(x, s_{q}\right)\right) \text { for each } i \in I_{p} .
$$

By using this notation $c^{(i)}(x)$, the authors introduced the notation $\hat{c}^{(i)}(x)$ to refer to the reordered vector of the vector $c^{(i)}(x)$ in nonincreasing way, that is,

$$
\hat{c}^{(i)}(x):=\left(\hat{c}_{1}^{(i)}(x), \hat{c}_{2}^{(i)}(x), \ldots, \hat{c}_{q}^{(i)}(x)\right),
$$

where $\hat{c}_{j}^{(i)}(x)=\operatorname{sort}_{j}\left(c^{(i)}(x)\right)$, for all $j \in I_{q}$. Consequently, for each $x \in \mathbb{R}^{n}$ and $j \in I_{q}$, they introduced the worst performance vector, worst $_{j}(f(x, \mathcal{U}))$, by

$$
\text { worst }_{j}(f(x, \mathcal{U})):=\left(\hat{c}_{j}^{(1)}(x), \hat{c}_{j}^{(2)}(x), \ldots, \hat{c}_{j}^{(p)}(x)\right) .
$$

Subsequently, the vector $\left(\hat{c}_{1}^{*}, \hat{c}_{2}^{*}, \ldots, \hat{c}_{q}^{*}\right)=: \hat{c}^{*} \in \mathbb{R}^{p \times q}$ will be called the reference point of the problem $\mathcal{M P}(\mathcal{U})$ if

$$
\hat{c}_{j}^{*}=\inf _{\text {with lex }}\left\{\text { worst }_{j}(f(x, \mathcal{U})) \mid x \in X\right\},
$$

for each $j \in I_{q}$.

Here, the lexicographic tolerable robust solution concept which was introduced by Boriwan et al. [2] is presented.

Definition 2.1. Let $\mathcal{M P}(\mathcal{U})$ be an uncertain multi-objective optimization problem together with the reference point $\left(\hat{c}_{1}^{*}, \hat{c}_{2}^{*}, \ldots, \hat{c}_{q}^{*}\right)=: \hat{c}^{*} \in \mathbb{R}^{p \times q}$. For each $\alpha:=\left(\alpha_{1}, \alpha_{2}, \ldots, \alpha_{q}\right) \in$ $[0, \infty)^{p \times q}$, the set of lexicographic tolerable robust solutions with respect to the tolerance threshold $\alpha$, which will be denoted by $\operatorname{LRS}(\alpha)$, is

$$
\operatorname{LRS}(\alpha):=\bigcap_{j=1}^{q} A_{j}^{\alpha_{j}},
$$


where $A_{j}^{\alpha_{j}}:=\left\{x \in X \mid\right.$ worst $\left._{j}(f(x, \mathcal{U})) \in\left(\hat{c}_{j}^{*}+\alpha_{j}\right)-\mathbb{R}_{\succsim}^{p}\right\}$.

Remark 2.1. (i) When $p=1$, that is we are concerned with an uncertain single objective optimization problem, the concept of lexicographic tolerable robust solution in Definition 2.1 is identical to the lexicographic $\alpha$-robust solution which was introduced by Kalaï et al. [9].

(ii) When $|\mathcal{U}|=1$, this means that we are dealing with deterministic multi-objective optimization problems. If $\alpha$ is the zero vector, then the solution concept in Definition 2.1 is nothing but the classical lexicographic solution concept, see [5] for instance. Notice that there is a concept so-called TOPSIS that also involved the concept of the reference point (which is called the ideal point in [7]). However, the TOPSIS method and the lexicographic tolerable robust solution method do have significant differences in computation of the reference point. Indeed, according to the lexicographic tolerable robust solution concept, the reference point is derived by using the lexicographic order relation in comparing the vector in the image space. While, the TOPSIS method, the reference point will be computed by considering each respective component of the objective function separately by regardless the priority levels in the objective function.

\section{PROperties of $L R S(\alpha)$}

Now the important properties of the set $L R S(\alpha)$ will be studied and interpreted. We begin this section by recalling a result in Kalaï et al. [9], which will be used to prove the important proposition on a relationship between each component of objective function under all scenarios of an alternative solution and the worst performance vector.

Lemma 3.1. [9] Given an uncertain single objective optimization problem and alternative solutions $x, y \in X$. If $f\left(x, s_{j}\right) \leqslant f\left(y, s_{j}\right)$, for each $s_{j} \in \mathcal{U}$, then

$$
\hat{c}_{j}(x) \leqslant \hat{c}_{j}(y) \text {, for all } j \in I_{q},
$$

where $f: \mathbb{R}^{n} \times \mathcal{U} \rightarrow \mathbb{R}$ and the function $\hat{c}$ is defined as in (2.5).

The following fact immediately follows from the Lemma 3.1.

Proposition 3.1. Given $\mathcal{M P}(\mathcal{U})$ be an uncertain multi-objective optimization problem with the uncertainty set $\mathcal{U}=\left\{s_{1}, s_{2}, \ldots, s_{q}\right\}$. If $x$ and $y$ are alternative solutions in $X$ and satisfy the relation

$$
c^{(i)}(x) \precsim c^{(i)}(y) \text {, for all } i \in I_{p}
$$

then

$$
\text { worst }_{j}(f(x, \mathcal{U})) \precsim \text { worst }_{j}(f(y, \mathcal{U})), \text { for all } j \in I_{q} \text {. }
$$

Proof. From the relation (3.7), by applying Lemma 3.1 to each value objective function $f_{i}$, we have

$$
\hat{c}^{(i)}(x) \precsim \hat{c}^{(i)}(y),
$$

for all $i \in I_{p}$. This immediately implies that

$$
\text { worst }_{j}(f(x, \mathcal{U})) \precsim \text { worst }_{j}(f(y, \mathcal{U})) \text {, for all } j \in I_{q} \text {. }
$$

Property 3.1. [Dominance] Let $x \in L R S(\alpha)$. If $y \in X$ satisfies

$$
c^{(i)}(y) \precsim c^{(i)}(x) \text {, for all } i \in I_{p},
$$

then $y \in \operatorname{LRS}(\alpha)$. 
Proof. The proof is directly followed from Proposition 3.1 and Definition 2.1.

Interpretation 3.2. The Property 3.1 stipulates that, if there is another alternative solution in a feasible set $X$ which dominates a robust solution under all scenarios, then it must be a robust solution.

The following fact leads us to some important results.

Lemma 3.2. Let $X \subseteq \mathbb{R}^{n}$ and $x \in X$. If $x \precsim \inf _{\text {with lex }} X$, then $x=\inf _{\text {with lex }} X$.

Proof. For sake of simplicity, we write $y^{*}:=\inf _{\text {with lex }} X$. Notice that from $y^{*}$ is the infimum with respect to lexicographic order relation and $x \in X$, it imply that $y^{*} \leq_{l e x} x$. Suppose that $x \neq y^{*}$. Then, there exists at least one $k \in I_{n}$ such that $x_{k} \neq y_{k}^{*}$. Defining $m:=$ $\min \left\{k \mid x_{k} \neq y_{k}^{*}\right\}$. From $x \precsim y^{*}$, it would follow that

$$
x_{i}=y_{i}^{*}, \text { for all } i=1,2, \ldots, m-1 \text { and } x_{m}<y_{m}^{*} .
$$

This implies that $x<_{\text {lex }} y^{*}$. This leads to a contradiction with the assumption that $y^{*}$ being the infimum. Hence, we can conclude that $x=y^{*}$.

Next, we will consider the non preference property for the set $\operatorname{LRS}(\alpha)$. To do so, we will consider the following binary relation with respect to a vector $\alpha$ under cone $\mathbb{R}_{\succcurlyeq}^{p}$. Let $z$ and $z^{\prime}$ be vectors in $\mathbb{R}^{p}$, and $\alpha \in \mathbb{R}_{\succcurlyeq}^{p}$, the relation $\rfloor_{\mathbb{R}_{\succcurlyeq}^{p}}^{\alpha}$ on $\mathbb{R}_{\succcurlyeq}^{p}$ is defined as follows:

$$
z \leqq \underset{\mathbb{R}_{\succcurlyeq}^{p}}{\alpha} z^{\prime} \Leftrightarrow z^{\prime}-\alpha \in z+\mathbb{R}_{\succcurlyeq}^{p} .
$$

Proposition 3.2. [Non Preference] Let $\alpha:=\left(\alpha_{1}, \alpha_{2}, \ldots, \alpha_{q}\right) \in[0, \infty)^{p \times q}$. If $x \in L R S(\alpha)$ and $z \notin L R S(\alpha)$, then for each $j \in I_{q}$, we have

$$
\text { worst }_{j}(f(z, \mathcal{U})) \quad \varliminf_{\mathbb{R}_{\succcurlyeq}^{p}}^{\alpha_{j}} \text { worst }_{j}(f(x, \mathcal{U})) .
$$

Proof. Let $j \in I_{q}$. Clearly, if worst $_{j}(f(z, \mathcal{U}))$ and worst $_{j}(f(x, \mathcal{U}))$ are not comparable, then the conclusion is obtained.

Now, we consider the case that worst $_{j}(f(z, \mathcal{U}))$ and worst $_{j}(f(x, \mathcal{U}))$ are comparable. Supposing on the contrary that

$$
\text { worst }_{j}(f(z, \mathcal{U})) \leqq_{\mathbb{R}_{\succcurlyeq}^{p}}^{\alpha_{j}} \text { worst }_{j}(f(x, \mathcal{U})) .
$$

Thus, by the definition of $\varliminf_{\mathbb{R}_{\succcurlyeq}^{p}}^{\alpha_{j}}$, we would get

$$
\text { worst }_{j}(f(z, \mathcal{U})) \preccurlyeq \text { worst }_{j}(f(x, \mathcal{U}))-\alpha_{j} \text { and } \text { worst }_{j}(f(z, \mathcal{U})) \neq \text { worst }_{j}\left(f(x, \mathcal{U})-\alpha_{j}\right. \text {. }
$$

Subsequently, since $x \in L R S(\alpha)$, we see that

$$
\text { worst }_{j}(f(z, \mathcal{U})) \preccurlyeq \text { worst }_{j}(f(x, \mathcal{U}))-\alpha_{j} \precsim\left(\hat{c}_{j}^{*}+\alpha_{j}\right)-\alpha_{j}=\hat{c}_{j}^{*} \text {. }
$$

Then, from the equation (3.10) together with the definition of the reference point $\hat{c}_{j}^{*}$ and Lemma 3.2, we could get

$$
\text { worst }_{j}(f(z, \mathcal{U}))=\hat{c}_{j}^{*} \text {. }
$$

In view of (3.10) and (3.11), we obtain that

$$
\text { worst }_{j}(f(z, \mathcal{U}))=\text { worst }_{j}(f(x, \mathcal{U}))-\alpha_{j},
$$

which leads to a contradiction with the assumption that

$$
\text { worst }_{j}(f(z, \mathcal{U})) \neq \text { worst }_{j}\left(f(x, \mathcal{U})-\alpha_{j}\right. \text {. }
$$

Therefore,

$$
\text { worst }_{j}(f(z, \mathcal{U})) \underset{\mathbb{R}_{\succcurlyeq}^{p}}{\alpha_{j}} \text { worst }_{j}(f(x, \mathcal{U})) \text { for all } j \in I_{q},
$$


this completes the proof.

Interpretation 3.3. Here, the non preference property means that there is no preferable solution being outside the set $\operatorname{LRS}(\alpha)$ with respect to the operator worst $(f(\cdot, \mathcal{U}))$, for each $j \in I_{q}$ and tolerance threshold $\alpha \in \mathbb{R}_{\succcurlyeq}^{p}$.

The following proposition will be concerned with the stability of the solution set $L R S(\alpha)$.

Proposition 3.3. [Stability] For any $x, x^{\prime} \in L R S(\alpha)$ where $\alpha:=\left(\alpha_{1}, \alpha_{2}, \ldots, \alpha_{q}\right) \in[0, \infty)^{p \times q}$, we have

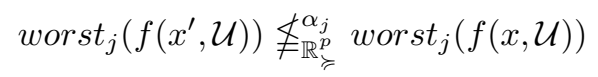

and

$$
\text { worst }_{j}(f(x, \mathcal{U})) \underset{\mathbb{R}_{\succcurlyeq}^{p}}{\alpha_{j}} \text { worst }_{j}\left(f\left(x^{\prime}, \mathcal{U}\right)\right)
$$

for all $j \in I_{q}$.

Proof. Let $j \in I_{q}$ be fixed. Suppose on the contrary that

$$
\text { worst }_{j}\left(f\left(x^{\prime}, \mathcal{U}\right)\right) \varliminf_{\mathbb{R}_{\succcurlyeq}^{p}}^{\alpha_{j}} \text { worst }_{j}(f(x, \mathcal{U}))
$$

or

$$
\text { worst }_{j}(f(x, \mathcal{U})) \varliminf_{\mathbb{R}_{\succcurlyeq}^{p}}^{\alpha_{j}} \text { worst }_{j}\left(f\left(x^{\prime}, \mathcal{U}\right)\right) .
$$

We may assume that worst $_{j}\left(f\left(x^{\prime}, \mathcal{U}\right)\right) \leqq_{\mathbb{R}_{\succcurlyeq}^{p}}^{\alpha_{j}}$ worst $_{j}(f(x, \mathcal{U}))$. From the definition of notation $\varliminf_{\mathbb{R}_{\succcurlyeq}^{p}}^{\alpha_{j}}$, we see that

$$
\text { worst }_{j}\left(f\left(x^{\prime}, \mathcal{U}\right)\right) \preccurlyeq \text { worst }_{j}(f(x, \mathcal{U}))-\alpha_{j} \text {. }
$$

Consequently, since $x \in L R S(\alpha)$, we get that

$$
\text { worst }_{j}\left(f\left(x^{\prime}, \mathcal{U}\right)\right) \preccurlyeq \operatorname{worst}_{j}(f(x, \mathcal{U}))-\alpha_{j} \precsim\left(\hat{c}_{j}^{*}+\alpha_{j}\right)-\alpha_{j}=\hat{c}_{j}^{*} .
$$

But (3.12) means that worst ${ }_{j}\left(f\left(x^{\prime}, \mathcal{U}\right)\right)$ is less than or equal to $\hat{c}_{j}^{*}$ in every component and there is at least one component of worst $_{j}\left(f\left(x^{\prime}, \mathcal{U}\right)\right)$ which is strictly less than $\hat{c}_{j}^{*}$. This is a contradiction to the definition of $\hat{c}_{j}^{*}$ being the infimum of set $\left\{\right.$ worst $\left._{j}(f(x, \mathcal{U})) \mid x \in X\right\}$. Therefore, we have

$$
\text { worst }_{j}\left(f\left(x^{\prime}, \mathcal{U}\right)\right) \oiint_{\mathbb{R}_{\succcurlyeq}^{p}}^{\alpha_{j}} \text { worst }_{j}(f(x, \mathcal{U})) .
$$

We can obtain the conclusion for the case worst $_{j}(f(x, \mathcal{U})) \leqq_{\mathbb{R}_{\succcurlyeq}^{p}}^{\alpha_{j}}$ worst $_{j}\left(f\left(x^{\prime}, \mathcal{U}\right)\right)$ by following analogously the proof of case worst $_{j}\left(f\left(x^{\prime}, \mathcal{U}\right)\right) \varliminf_{\mathbb{R}_{\succcurlyeq}^{p}}^{\alpha_{j}}$ worst $_{j}(f(x, \mathcal{U}))$.

Interpretation 3.4. Proposition 3.3 shows that there is no preferable solution among elements in the set $L R S(\alpha)$ via considering the preference defined by the order relation $\varliminf_{\mathbb{R}_{\succcurlyeq}^{p}}^{\alpha}$ on $\mathbb{R}_{\succcurlyeq}^{p}$.

\section{FURTHER DISCUSSION}

In this section, we consider the links between the lexicographic tolerable robust solution and the set-based minmax robust efficiency, which was introduced by Ehrgott et al. [4]. To do this, we will denote the set of all possible objective vectors under all scenarios of each alternative solution $x \in X$ with the following notation:

$$
f_{\mathcal{U}}(x):=\{f(x, s): s \in \mathcal{U}\} \subseteq \mathbb{R}^{p}
$$

We now recall the formal definition of set-based robust efficiency [4]. 
Definition 4.2. [4] Given an uncertain multi-objective optimization problem $\mathcal{M P}(\mathcal{U})$. An alternative solution $\hat{x} \in X$ is called a set-based minmax robust efficient solution if there is no $x \in X \backslash\{\hat{x}\}$ such that

$$
f_{\mathcal{U}}(x) \subseteq f_{\mathcal{U}}(\hat{x})-\mathbb{R}_{\succcurlyeq}^{p}
$$

Notice that by replacing the cone $\mathbb{R}_{\succcurlyeq}^{p}$ with $\mathbb{R}_{\succsim}^{p}$ or $\mathbb{R}_{\succ}^{p}$ in Definition 4.2 , the alternative solution $\hat{x}$ is called $a$ set-based minmax robust strictly efficient solution or a set-based minmax robust weakly efficient solution, respectively.

Remark 4.2. We point out that the underlying ideas of the lexicographic tolerable robust solution concept and the set-based minmax robust efficiency are not identical. According to their own solution concepts, the corresponding solutions with respect to each solution concept do have the particularity structure on applying to the different practical problems. Indeed, the solution is of which corresponding to the lexicographic tolerable robust solution concept will provide the priority levels on the objective function, while the set-based minmax robust efficiency concept will provide a good trade-off for all objective function. For example, the lexicographic tolerable robust solution concept have successfully been applied to the problem of water resources management planning as the priories on each objective was provided by the decision makers [2], while the set-based robust efficiency concept was employed in the design of sustainable energy system to identify the designs of the problem with a good trade-off between economic and ecological criteria, for more information see [11].

To present the results on relationships between the lexicographic tolerable robust solution and the set-based minmax robust efficiency, we recall the following supplementary result.

Lemma 4.3. [4] Given an uncertain multi-objective optimization problem $\mathcal{M P}(\mathcal{U})$. Then, the following statements hold.

(a) For all $x^{\prime}, \bar{x} \in X$,

$$
f_{\mathcal{U}}\left(x^{\prime}\right) \subseteq f_{\mathcal{U}}(\bar{x})-\mathbb{R}_{[\succsim / \succcurlyeq / \succ]}^{p} \Longleftrightarrow f_{\mathcal{U}}\left(x^{\prime}\right)-\mathbb{R}_{\succsim}^{p} \subseteq f_{\mathcal{U}}(\bar{x})-\mathbb{R}_{[\succsim / \succcurlyeq / \succ]}^{p} .
$$

(b) For all $x^{\prime}, \bar{x} \in X$,

$f_{\mathcal{U}}\left(x^{\prime}\right)-\mathbb{R}_{\succsim}^{p} \subseteq f_{\mathcal{U}}(\bar{x})-\mathbb{R}_{[\succsim / \succcurlyeq / \succ]}^{p} \Longleftrightarrow \forall s \in \mathcal{U} \exists s^{\prime} \in \mathcal{U}: f\left(x^{\prime}, s\right)[\precsim / \precsim / \prec] f\left(\bar{x}, s^{\prime}\right)$.

(c) For all $x^{\prime}, \bar{x} \in X$,

$$
f_{\mathcal{U}}\left(x^{\prime}\right)-\mathbb{R}_{\succsim}^{p} \subseteq f_{\mathcal{U}}(\bar{x})-\mathbb{R}_{\succsim}^{p} \Rightarrow \sup _{s \in \mathcal{U}} f_{i}\left(x^{\prime}, s\right) \leqslant \sup _{s^{\prime} \in \mathcal{U}} f_{i}\left(\bar{x}, s^{\prime}\right),
$$

for all $i \in I_{p}$.

(d) If $\max _{s \in \mathcal{U}} f_{i}(x, s)$ exists, for all $x \in X$ and $i \in I_{p}$, then for all $x^{\prime}, \bar{x} \in X$,

$$
f_{\mathcal{U}}\left(x^{\prime}\right)-\mathbb{R}_{\succsim}^{p} \subseteq f_{\mathcal{U}}(\bar{x})-\mathbb{R}_{\succ}^{p} \Rightarrow \max _{s \in \mathcal{U}} f_{i}\left(x^{\prime}, s\right)<\max _{s^{\prime} \in \mathcal{U}} f_{i}\left(\bar{x}, s^{\prime}\right),
$$

for all $i \in I_{p}$.

The following result presents the technique for finding the set-based robust efficiency via the lexicographic tolerable robust solution idea.

Theorem 4.1. Let $\mathcal{M P}(\mathcal{U})$ be an uncertain multi-objective optimization problem with the reference point $\left(\hat{c}_{1}^{*}, \hat{c}_{2}^{*}, \ldots, \hat{c}_{q}^{*}\right)=: \hat{c}^{*} \in \mathbb{R}^{p \times q}$ and 0 the zero vector in $\mathbb{R}^{p}$. If $\hat{x} \in A_{1}^{0}$ and $A_{1}^{0}$ is defined as in Definition 2.1, then $\hat{x}$ is a set-based robust weakly efficient solution for the problem $\mathcal{M P}(\mathcal{U})$. 
Proof. Since $\hat{x} \in A_{1}^{0}$, we have that

$$
\operatorname{worst}_{1}(f(\hat{x}, \mathcal{U})) \precsim \hat{c}_{1}^{*} .
$$

By the definition of reference point $\hat{c}_{1}^{*}$ together with Lemma 3.2, it follows that

$$
\text { worst }_{1}(f(\hat{x}, \mathcal{U}))=\hat{c}_{1}^{*} \text {. }
$$

Suppose that $\hat{x}$ is not a set-based weakly robust efficient solution. Then, there exists $x \in X \backslash\{\hat{x}\}$ such that

$$
f_{\mathcal{U}}(x) \subseteq f_{\mathcal{U}}(\hat{x})-\mathbb{R}_{\succ}^{p} .
$$

By applying the items (a) and (d) of Lemma 4.3, we have that

$$
\max _{s \in \mathcal{U}} f_{i}(x, s)<\max _{s \in \mathcal{U}} f_{i}(\hat{x}, s), \text { for all } i \in I_{p} .
$$

By the definition of $\hat{c}_{1}^{(i)}(\cdot)$ and definition of worst $_{1}(f(\cdot, \mathcal{U}))$, it imply that

$$
\text { worst }_{1}(f(x, \mathcal{U})) \preccurlyeq \text { worst }_{1}(f(\hat{x}, \mathcal{U}))=\hat{c}_{1}^{*} \text {. }
$$

This leads to a contradiction with $\hat{c}_{1}^{*}$ being the infimum of set $\left\{\right.$ worst $\left._{1}(f(x, \mathcal{U})) \mid x \in X\right\}$. Therefore $\hat{x}$ is a set-based robust weakly efficient solution for the problem $\mathcal{M P}(\mathcal{U})$.

The following result provides a sufficient condition on a technique for finding the setbased robust strictly efficient solution for the problem $\mathcal{M P}(\mathcal{U})$.

Theorem 4.2. Let $\mathcal{M P}(\mathcal{U})$ be an uncertain multi-objective optimization problem together with reference point $\left(\hat{c}_{1}^{*}, \hat{c}_{2}^{*}, \ldots, \hat{c}_{q}^{*}\right)=: \hat{c}^{*} \in \mathbb{R}^{p \times q}$. Let $\alpha_{1}=(\alpha, \alpha, \ldots, \alpha) \in \mathbb{R}_{\succsim}^{p}$. If $A_{1}^{\alpha_{1}}=\{\hat{x}\}$ and $A_{1}^{\alpha_{1}}$ is defined as in Definition 2.1, then $\hat{x}$ is a set-based minmax robust strictly efficient solution for the problem $\mathcal{M P}(\mathcal{U})$.

Proof. From $\hat{x} \in A_{1}^{\alpha_{1}}$, it follows that

$$
\text { worst }_{1}(f(\hat{x}, \mathcal{U})) \in\left(\hat{c}_{1}^{*}+\alpha_{1}\right)-\mathbb{R}_{\succsim}^{p} \text {. }
$$

This means that,

$$
\operatorname{worst}_{1}(f(\hat{x}, \mathcal{U})) \precsim \hat{c}_{1}^{*}+\alpha_{1} \text {. }
$$

For each $j \in I_{q}$, we write $\hat{c}_{j}^{*}=\left(\hat{c}_{j}^{*(1)}, \hat{c}_{j}^{*(2)}, \ldots, \hat{c}_{j}^{*(p)}\right)$. It follows that,

$$
\hat{c}_{1}^{(i)}(\hat{x}) \leqslant \hat{c}_{1}^{*(i)}+\alpha, \text { for all } i \in I_{p}
$$

Suppose on the contrary, that $\hat{x}$ is not set-based minmax robust strictly efficient for the problem $\mathcal{M P}(\mathcal{U})$. By the Definition 4.2, there is $x \in X \backslash\{\hat{x}\}$ such that

$$
f_{\mathcal{U}}(x) \subseteq f_{\mathcal{U}}(\hat{x})-\mathbb{R}_{\succsim}^{p}
$$

By the items (a), (b) and (c) of Lamma 4.3, we obtain that

$$
\max _{s \in \mathcal{U}} f_{i}(x, s) \leqslant \max _{s \in \mathcal{U}} f_{i}(\hat{x}, s), \text { for all } i \in I_{p} .
$$

We note that for each $i \in I_{p}$, by the definition of $\hat{c}_{1}^{(i)}(\cdot)$, we have that

$$
\hat{c}_{1}^{(i)}(x)=\max _{s \in \mathcal{U}} f_{i}(x, s), \text { for all } x \in X .
$$

From the equations (4.19) and (4.20), it follow that

$$
\text { worst }_{1}(f(x, \mathcal{U})) \precsim \text { worst }_{1}(f(\hat{x}, \mathcal{U})) \text {. }
$$

Thus, from the equations (4.18) and (4.21), we must have that $x$ is an another element in $A_{1}^{\alpha_{1}}$. This leads to a contradiction with the assumption that $\hat{x}$ being the unique element in 
$A_{1}^{\alpha_{1}}$. Therefore, $\hat{x}$ is a set-based minmax robust strictly efficient solution for the problem $\mathcal{M P}(\mathcal{U})$.

Remark 4.3. To find a threshold $\alpha_{1}$ that the set $A_{1}^{\alpha_{1}}$ is a singleton, is not so difficult. This is because, if we start with tolerance threshold $\alpha_{1}$, then one can be refined to obtain the desirable singleton set $A_{1}^{\alpha_{1}^{n e w}}$, where the updating tolerance threshold $\alpha_{1}^{\text {new }}$ can be computed via the method presented by Boriwan et al. in [2]. Nevertheless, there is only one situation that even we refine the tolerance threshold $\alpha_{1}$, the corresponding set $A_{1}^{\alpha_{1}^{\text {new }}}$ still not singleton, that is there are several alternative solutions providing the same worst performance vector worst $_{1}(f(\cdot, \mathcal{U}))$.

The following example shows a situation that $A_{1}^{\alpha_{1}}$ is not a singleton set for any choice of $\alpha_{1}$.

Example 4.1. Let $X=\left\{x_{1}, x_{2}, x_{3}, x_{4}\right\}$ be the considered feasible set. The information about vector-valued function $f$ for each alternative solution $x_{i}$ estimated under two possible scenarios $s_{1}$ and $s_{2}$ are shown in Table 1 . Consequently, the sort function $\hat{c}^{(i)}(\cdot)$ of each component function $f_{i}$ and also the $j^{\text {th }}$ worst performance vector of each alternative solution $x_{i}$ are provided in Table 2. Thus, it follows that $A_{1}^{\alpha_{1}}=\left\{x_{2}, x_{3}, x_{4}\right\}$, for all $\alpha_{1}:=(\alpha, \alpha) \in \mathbb{R}_{\succsim}^{2}$.

Moreover, according to the Definition 4.2, we can check that the set-based minmax robust efficiency solution set is $\left\{x_{1}, x_{4}\right\}$. This means, for any choice of $\alpha_{1}$, the set $A_{1}^{\alpha_{1}}$ is not a subset of set-based minmax robust efficiency solution set. Furthermore, by applying the method of computing the smallest tolerance threshold which can be guaranteed the nonemptyness of the lexicographic tolerable robust solution concept in [2], we found that the such solution set is $\operatorname{LRS}\left(\alpha^{*}\right)=\left\{x_{3}\right\}$ with $\alpha^{*}:=((1,1),(1,1))$. This shows that the solution sets of those related to lexicographic tolerable robust solution concept and setbased minmax robust efficiency solution concept can be (extremely) different.

\begin{tabular}{ccccc}
\hline & \multicolumn{4}{c}{ Objective Function } \\
\cline { 2 - 5 } Alternatives & $\boldsymbol{f}_{\mathbf{1}}\left(\cdot, \boldsymbol{s}_{\mathbf{1}}\right)$ & $\boldsymbol{f}_{\mathbf{1}}\left(\cdot, \boldsymbol{s}_{\mathbf{2}}\right)$ & $\boldsymbol{f}_{\mathbf{2}}\left(\cdot, \boldsymbol{s}_{\mathbf{1}}\right)$ & $\boldsymbol{f}_{\mathbf{2}}\left(\cdot, \boldsymbol{s}_{\mathbf{2}}\right)$ \\
\hline$x_{1}$ & 4 & 11 & 14 & 4 \\
$x_{2}$ & 10 & 10 & 11 & 7 \\
$x_{3}$ & 10 & 5 & 11 & 4 \\
$x_{4}$ & 10 & 6 & 7 & 11 \\
\hline
\end{tabular}

TABLE 1 . The objective function $f=\left(f_{1}, f_{2}\right)$ for each alternative solution $x_{k}$ under all scenarios $s_{j}$.

\begin{tabular}{ccccc}
\hline Alternatives & $\hat{\boldsymbol{c}}^{(\mathbf{1})}(\cdot)$ & $\hat{\boldsymbol{c}}^{(\mathbf{2})}(\cdot)$ & worst $_{\mathbf{1}}(\boldsymbol{f}(\cdot, \mathcal{U}))$ & wor $_{\boldsymbol{2}}(\boldsymbol{f}(\cdot, \mathcal{U}))$ \\
\hline$x_{1}$ & $(11,4)$ & $(14,4)$ & $(11,14)$ & $(4,4)$ \\
$x_{2}$ & $(10,10)$ & $(11,7)$ & $(10,11)$ & $(10,7)$ \\
$x_{3}$ & $(10,5)$ & $(11,4)$ & $(10,11)$ & $(5,4)$ \\
$x_{4}$ & $(10,6)$ & $(11,7)$ & $(10,11)$ & $(6,7)$ \\
\hline$\hat{c}_{j}^{*}$ & & & $\hat{c}_{1}^{*}=(10,11)$ & $\hat{c}_{2}^{*}=(4,4)$ \\
\hline
\end{tabular}

TABLE 2. The function $\hat{c}^{(i)}(\cdot)$ and wor $_{s}(f(\cdot, \mathcal{U}))$. 


\section{CONCLUSION}

In this paper, we presented the important properties of the lexicographic tolerable robust solution which was introduced by Boriwan et al. [2]. Furthermore, we considered the links between the lexicographic tolerable robust solution concept [2] and the setbased minmax robust efficient solution concept [4]. Moreover, we provide a technique for computing an element of set-based minmax robust efficient solution by using the lexicographic tolerable robust solution idea.

Acknowledgements. The authors are extremely thankful to the anonymous referees and the editor for providing valuable suggestions to improve the paper to its current form greatly. This research was supported by the Thailand Research Fund through the Royal Golden Jubilee Ph. D. Program Grant No. PHD/0046/2559 and JSPS KAKENHI Grant Number 19K03637

\section{REFERENCES}

[1] Ben-tal, A.; Nemirovski, A. Robust convex optimization. Mathematics of Operations Research. 23 (1998), 769-805.

[2] Boriwan, P.; Ehrgott, M.; Kuroiwa, D.; Petrot, N. The lexicographic tolerable robustness concept for uncertain multi-objective optimization problems: a study on water resources management. Sustainability. 12 (2020), no. 18, article number 7582.

[3] Burke, E. K.; Causmaecker, P. D.; Maere, G. D.; Mulder, J.; Paelinck, M.; Berghe, G. V. A multi-objective approach for robust airline scheduling. Computers and Operation Research. 37 (2010), no. 5, 822-832.

[4] Ehrgott, M.; Ide, J.; Schöbel, A. Minmax robustness for multi-objective optimization problems. European Journal of Operational Research. 239 (2014), no. 1, 17-31.

[5] Ehrgott, M. Multicriteria optimization. Second edition. Germany: Springer Berlin Heidelberg, 2005.

[6] Goerigk, M.; Schmidt, M.; Schöbel, A.; Knoth, M.; Müller-Hannemann, M. The Price of Strict and Light Robustness in Timetable Information. Transportation Science. 48 (2014), no. 2, 225-242.

[7] Hwang, C. L.; Yoon, K. Multiple Attribute Decision Making: Methods and Applications A State-of-the-Art Survey (Lecture Notes in Economics and Mathematical Systems). First edition. New York: Springer-Verlag, 1981.

[8] Ide, J.; Schöbel, A. Robustness for uncertain multi-objective optimization: a survey and analysis of different concepts. OR Spectrum. 38 (2016), 235-271.

[9] Kalaï, R.; Lamboray, C.; Vanderpooten, D. Lexicographic $\alpha$-robustness: an alternative to min-max criteria. European Journal of Operational Research. 220 (2012), 722-728.

[10] Kuroiwa, D.; Lee, G. M. On robust multiobjective optimization. Vietnam Journal of Mathematics. 40 (2012), 305-317.

[11] Majewski, D. E.; Wirtz, M.; Lampe, M.; Bardow, A. Robust multi-objective optimization for sustainable design of distributed energy supply systems. Computers and Chemical Engineering. 102 (2017), 26-39.

[12] Perelman, L.; Housh, M.; Ostfeld, A. Robust optimization for water distribution systems least cost design. Water Resources Resarch. 49 (2013), 6795-6809.

[13] Prons-Prats, J.; Bugeda, G.; Zarate, F.; Oñate, E.; Periaux, J. Applying multi-objective robust design optimization procedure to the route planning of commercial aircraft. Computational Methods and Models for Transport. Computational Methods in Applied Sciences. 45 (2018), 147-167.

[14] Soyster, A. Convex programming with set-inclusive constraints and applications to inexact linear programming. Oper. Res. 21 (1973), 1154-1157.

${ }^{1}$ Department of Mathematics, Faculty of Science

NARESUAN UNIVERSITY, PHITSANUlOK 65000, THAILAND

Email address: boriwanp_1990@hotmail.com

Email address: narinp@nu.ac.th

2 Department of Mathematics, Faculty of SCiEnCE ANd Engineering

SHIMANE UNIVERSITY, SHIMANE, 690-0823, JAPAN

Email address: kuroiwa@riko.shimane-u.ac.jp

${ }^{3}$ Research Center of Excellence in Nonlinear ANAlysis ANd Optimization

FACUlty of SCIENCE, NARESUAN University, PhitsanuloK 65000, Thailand

Email address: narinpenu.ac.th 